\title{
L'approccio al marketing e alla comunicazione nelle piccole e medie imprese: una ricerca empirica in Italia e UK
}

\author{
CARMELA TuCCILlO
}

\begin{abstract}
Obiettivo del paper: La ricerca di natura descrittiva ha come obiettivi monitorare l'utilizzo delle leve di marketing e comunicazione nelle piccole e medie imprese (PMI) attraverso una comparazione cross-country Italia-Inghilterra e colmare un gap in letteratura.

Metodologia: Le due fasi della ricerca quantitativa e qualitativa hanno coinvolto un campione di 100 PMI nel periodo febbraio-novembre 2011. Il questionario allestito sulla base dei contributi rilevati in letteratura è stato somministrato attraverso una web-survey. Infine, la cluster analysis è servita a sintetizzare i risultati attraverso la profilazione di tre atteggiamenti tipo.

Risultati: Da una visione d'insieme emerge che le PMI rispetto al passato tendono ad utilizzare un più ampio spettro di attività di marketing e comunicazione ricorrendo a maggiori investimenti. Tuttavia, esistono delle differenziazioni nell'approccio adottato dettate da ragioni intrinseche all'impresa come il settore di appartenenza, la dimensione, ecc.

Limiti della ricerca: L'eterogeneità settoriale e dimensionale dei due campioni non consente un confronto diretto e un'accurata analisi comparativa. Inoltre, la ridotta numerosità dei campioni assunti a base dell'analisi non permette di generalizzare i risultati.

Implicazioni pratiche: I risvolti manageriali della ricerca sono utili a delineare strategie di sviluppo e valorizzazione delle PMI nel contesto europeo contribuendo al dibattito sulla necessità di un approccio alla pianificazione di marketing e comunicazione nelle PMI.

Originalità del paper: A differenza dei precedenti studi, il lavoro si avvale di una ricerca empirica finalizzata alla migliore comprensione, a livello internazionale, delle dinamiche intrinseche all'adozione o meno di specifiche attività di marketing $e$ comunicazione nelle PMI.
\end{abstract}

Parole chiave: marketing; comunicazione d'impresa; piccole e medie imprese

Purpose of the paper: Despite the rich literature concerning the small and medium enterprises (SMEs), few studies have investigated the role of marketing and communication in this type of firms. In order to fill this gap, the paper concerns an empirical research on the

Dottore di Ricerca in Marketing e Comunicazione (X Ciclo) - Università degli Studi di Salerno

e-mail: ctuccillo@unisa.it 
organization of the marketing and communication in the SMEs, through an analysis of Italian and English firms, useful to recognize similarities and differences between the two samples.

Methodology: In order to identify distinctive characteristics of marketing strategies in 100 small medium enterprises in Italy and UK, a web survey is carried out. Moreover, the cluster analysis categorizes three different typologies of firms with specific features.

Findings: The results of the survey show that SMEs are beginning to recognize the importance of marketing and communication with a massive use of the activities related to them. However, there are differences in the approach to marketing and communication dictated by intrinsic characteristics of these firms e.g. sector, size, etc. Empirical evidences suggest that smaller firms are less oriented to adopt marketing and communication activities.

Research limitations: Research limitations concern the little number of firms within the two samples, in fact, it is impossible to generalize the results.

Practical implications: This study contributes to outline strategies for the development of small and medium-sized enterprises in the European context and to emphasize the necessity to plan marketing and communication activities in the SMEs.

Originality of the paper: This paper compared to previous studies, explore marketing and communication strategies using an empirical research.

Key words: marketing; corporate communication; small and medium enterprises

\section{Introduzione}

Le piccole e medie imprese (PMI) rivestono un ruolo strutturalmente importantissimo negli assetti produttivi di tutte le economie nazionali progredite ${ }^{1}$ (Mattiacci e Ceccotti, 2005). In particolare, in Italia, come è noto, la struttura produttiva rimane caratterizzata dalla fortissima presenza delle PMI, motore dell'economia nazionale.

Lo studio della letteratura ha evidenziato le enormi e profonde differenze che queste imprese presentano rispetto a quelle di più grande dimensione (Carson, 1985; Carson e Cromie, 1989; Carson, 1990; Carson, 1993; Carson et al., 1995; Carson e Coviello, 1996; Fillis, 2002; O’Dwyer et al., 2009). Le piccole e medie imprese mostrano tratti e caratteristiche di unicità, difficilmente paragonabili alle altre organizzazioni. Esse, infatti, risultano caratterizzate dalla marcata presenza della figura dell'imprenditore, che risulta coinvolto in tutte le attività aziendali; da una struttura organizzativa semplice, di tipo prettamente funzionale e dall'assenza di formalizzate procedure di pianificazione aziendale (Steinhoff, 1978; Mendham e Bannock, 1982). Queste caratteristiche rendono la piccola e media impresa una realtà a sé stante, contraddistinta da valori, comportamenti, meccanismi di funzionamento e management style del tutto tipici e singolari (Carson e McCartanQuinn, 1995; Carson et al., 1998; Carson e Grant, 1998; Carson e Gilmore, 2000).

1 In base alla raccomandazione 2003/361/CE, sono definite piccole e medie imprese, le imprese che hanno meno di 250 occupati e un fatturato annuo non superiore a 50 milioni di euro, oppure un totale di bilancio annuo non superiore a 43 milioni di euro. 
Sebbene esista una ricchissima letteratura sulle piccole e medie imprese (PMI), questo lavoro si propone di colmare una lacuna degli studi manageriali sul rapporto tra PMI e marketing/communication, quella di risultare, in genere, molto teorici e poco basati su dati empirici. Per dare risposta a tale questione, si propone dopo un approfondimento dei principali contributi prodotti a livello nazionale ed internazionale, una ricerca di natura descrittiva che ha come obiettivo, quello di monitorare l'utilizzo delle leve di marketing e comunicazione nelle piccole e medie imprese (PMI) attraverso una comparazione cross-country Italia-Inghilterra, utile ad individuare similitudini e differenze nell'approccio adottato.

\section{II marketing e la comunicazione nelle PMI. I contributi in letteratura}

Come si può vedere dalla schematizzazione proposta (Tab. 1), la letteratura in materia di PMI implicitamente ed esplicitamente evidenzia che le piccole e medie imprese presentano caratteristiche specifiche e differenti dalle grandi organizzazioni (Kirby e Travis, 1995). In particolare, le differenze riguardano gli obiettivi aziendali, il management style e le pratiche di marketing e comunicazione adottate (O'Dwyer et al., 2009; Fillis, 2002; Carson e Cromie, 1989).

Tab. 1: I principali contributi sul marketing nelle PMI

\begin{tabular}{|c|c|}
\hline Autori & Contributi \\
\hline $\begin{array}{l}\text { Varaldo (1983); Cozzi } \\
\text { (1985); Carson e Cromie } \\
\text { (1989); Kirby and Travis } \\
\text { (1995); Thrassou e Vrontis } \\
\text { (2006) }\end{array}$ & $\begin{array}{l}\text { L'approccio di marketing è estraneo allo stile direzionale della } \\
\text { piccola impresa, perché "il marketing è qualcosa di più e di diverso } \\
\text { dal semplice adattamento reattivo al mercato, in quanto implica } \\
\text { una vera e propria azione sul mercato". }\end{array}$ \\
\hline $\begin{array}{l}\text { Marchini (1985); O'Dwyer et } \\
\text { al., (2009) }\end{array}$ & $\begin{array}{l}\text { "Le PMI si caratterizzano per la propensione di chi le governa di } \\
\text { fare strategia in modo inconsapevole". }\end{array}$ \\
\hline $\begin{array}{l}\text { Grandinetti (1989); Siu and } \\
\text { Kirby (1998) }\end{array}$ & $\begin{array}{l}\text { "Le PMI sono caratterizzate da una gestione del marketing mix } \\
\text { quasi inesistente". }\end{array}$ \\
\hline $\begin{array}{l}\text { Mauri (1997 e 2011); } \\
\text { McCartan-Quinn e Carson } \\
\text { (2003); } \\
\text { Wong e Merrilees (2008) }\end{array}$ & $\begin{array}{l}\text { "Il marketing che alcune PMI sviluppano è solo un } \\
\text { rimpicciolimento del marketing che si sviluppa nelle grandi } \\
\text { imprese ed organizzazioni; esiste un marketing specifico per le } \\
\text { piccole e medie imprese". }\end{array}$ \\
\hline $\begin{array}{l}\text { Mattiacci e Ceccotti (2005); } \\
\text { Hätönen e Ruokonen (2010) }\end{array}$ & $\begin{array}{l}\text { "Vi sono tutte le condizioni perché le due «rette parallele», PMl e } \\
\text { marketing, si vadano ad incontrare a causa del prodursi - } \\
\text { concomitante o alternativo - di accadimenti esogeni (inasprimento } \\
\text { dell'intensità concorrenziale) e/o endogeni (nuova dotazione di } \\
\text { risorse)". }\end{array}$ \\
\hline $\begin{array}{l}\text { Carson (1990); Fortezza } \\
\text { (2007) }\end{array}$ & $\begin{array}{l}\text { "Il marketing attuato dalle PMI è soprattutto ascrivibile a una } \\
\text { interpretazione riduttiva ed incompleta del marketing concept". }\end{array}$ \\
\hline $\begin{array}{l}\text { Pencarelli e Cioppi (2006); } \\
\text { Bell et al., (2007) }\end{array}$ & $\begin{array}{l}\text { "Il paradigma del marketing management applicato alle PMI deve } \\
\text { essere oggetto di un adeguato adattamento". }\end{array}$ \\
\hline $\begin{array}{l}\text { De Luca (2009); O’Dwyer } \\
\text { (2009) }\end{array}$ & $\begin{array}{l}\text { In sostanza, le PMI svolgono attività di marketing reattivo e non } \\
\text { proattivo, tattico e non strategico, empirico ed intuitivo, piuttosto } \\
\text { che strutturato". }\end{array}$ \\
\hline
\end{tabular}

Fonte: ns. elaborazione 
Generalmente, le attività di marketing e comunicazione adottate nelle PMI risultano essere azzardate, non strutturate e prive di processi formalizzati di pianificazione strategica (McCartan-Quinn e Carson, 2003; Reynolds, 2002; Siu e Kirby, 1998). Inoltre, queste attività risultano basate su una quotidiana risoluzione di problematiche emergenti (approccio reattivo-tattico) piuttosto che su una più rigorosa pianificazione (approccio proattivo-strategico) (Yin e Merrilees, 2008; Bhide, 1994; Scase e Goffee, 1987). I principi del marketing e della comunicazione trovano infatti, in tali imprese declinazioni del tutto particolari sia in termini strategici che operativi (Pacitto et al., 2007; Gilmore et al., 2006; Thrassou e Vrontis, 2006; Mattiacci e Ceccotti, 2005; Cozzi e Ferrero, 2000; Marchini, 1985; Vescovi e Gazzola, 2006; Carson, 1985). Inoltre, è evidente, l'assoluta centralità della figura dell'imprenditore responsabile di tutte le attività di marketing management dell'impresa di piccola dimensione (marketing imprenditoriale o entrepreneurship marketing) (Bell et al., 2007; Guercini, 2005; Simpson e Taylor, 2002; Hill, 2001a, 2001b; Carson et al., 1995). Le linee strategiche sono definite direttamente dalla proprietà e si basano su processi intuitivi ancora radicati sull'esperienza diretta e sulla conoscenza del proprietario/manager (Lilien et al., 1992). Inoltre, è stato da più studiosi rilevato che le piccole e medie imprese tendono ad avere un atteggiamento negativo verso il marketing, percependolo come un costo, guardando alla distribuzione e alla vendita come problemi incontrollabili, e credendo che l'applicazione di regole generali di marketing non sia funzionale alla risoluzione delle problematiche aziendali (Cohn e Lindbore, 1972). È stata anche osservata, una generale debolezza delle piccole e medie imprese attribuibile alla difficoltà di reperire personale esperto qualificato nei ruoli dedicati al marketing e alla comunicazione (Broom e Longenecker, 1983). Infatti, le piccole e medie imprese a differenza delle grandi per risolvere i problemi aziendali adottano tecniche di tipo pragmatico invece di reperire, coordinare e controllare specialisti di marketing (Schollhammer e Kuriloff, 1979).

In tema di comunicazione, le piccole e medie imprese manifestano più o meno consapevolmente la necessità di comunicare, in vista del perseguimento di determinati obiettivi (Invernizzi e Romenti, 2012, 2013; Corvi e Fiocca, 1996; Watzlawick et al., 1971): creare, sostenere e preservare relazioni; generare e mantenere fiducia; sviluppare conoscenza; sviluppare credibilità e reputazione; diffondere il valore agli stakeholder attraverso la condivisione del patrimonio intangibile d'impresa. Una comunicazione consapevole, unita ad un efficace governo delle relazioni con tutti i portatori d'interesse, migliora la fiducia, la reputazione e la credibilità strategica della piccola e media impresa e ne incrementa il patrimonio intangibile (Vecchiato, 2005). Emerge da questo quadro iniziale, un interesse crescente nei confronti della comunicazione, che deve essere definita e gestita in maniera coordinata e integrata anche nelle imprese di più piccola dimensione. La comunicazione oggi, dovrebbe ricoprire un ruolo attivo e strategico e non solo tattico all'interno dell'impresa di piccola dimensione, che in questo modo potrebbe meglio assicurarsi il raggiungimento degli obiettivi aziendali prefissati (Kitchen e Schulz, 2000; Invernizzi e Romenti, 2012, 2013; Siano et. al., 2013). 
Tuttavia, nella realtà operativa è facile appurare che molte piccole e medie imprese utilizzino in maniera riduttiva la leva della comunicazione aziendale.

In conclusione, si ritiene che le specifiche caratteristiche di queste imprese (mancanza di competenze specialistiche, impatto limitato sul mercato, dimensione ridotta, area operativa locale, centralità dell'imprenditore) costituiscano dei limiti all'adozione di specifiche attività di marketing e comunicazione (Hogarth-Scott et al., 1996; Kirby and Travis, 1995) (Tab. 2). In generale, prevale un atteggiamento adattivo o reattivo nei confronti del mercato di riferimento orientato all'efficienza produttiva e, conseguentemente, un orientamento parziale al marketing e alla comunicazione.

Tab. 2: Caratteristiche delle PMI e relative debolezze di marketing

\begin{tabular}{|c|l|}
\hline Caratteristiche delle PMI & \multicolumn{1}{|c|}{ Debolezze di marketing } \\
\hline Risorse limitate & $\begin{array}{l}\text { Contribuiscono a limitare le attività di marketing rispetto alle } \\
\text { grandi imprese concorrenti. }\end{array}$ \\
\hline $\begin{array}{c}\text { Mancanza di competenze } \\
\text { specialistiche }\end{array}$ & $\begin{array}{l}\text { Rappresentano un limite nelle piccole e medie imprese; } \\
\text { tradizionalmente il proprietario/manager coinvolto in tutte le } \\
\text { attività aziendali è sprovvisto di competenze specialistiche. }\end{array}$ \\
\hline Impatto limitato sul mercato & $\begin{array}{l}\text { Le PMl hanno meno ordini, meno clienti e meno dipendenti } \\
\text { delle grandi imprese e di conseguenza hanno un limitato potere } \\
\text { di mercato. }\end{array}$ \\
\hline Dimensione ridotta & $\begin{array}{l}\text { Contribuisce alla mancata adozione di un approccio strutturato } \\
\text { al marketing. }\end{array}$ \\
\hline Centralità dell'imprenditore & $\begin{array}{l}\text { L'imprenditore che non ha competenze specialistiche di } \\
\text { marketing, utilizza in maniera azzardata e senza alcun tipo di } \\
\text { coordinamento alcune leve del marketing mix. }\end{array}$ \\
\hline Area operativa locale & Scarsa attitudine alla concorrenza e vulnerabilità. \\
\hline Efficienza operativa & Orientamento alla produzione, scarsi investimenti in marketing. \\
\hline
\end{tabular}

Fonte: adattata da Carson, 1990

Come già detto, emerge con forza il ruolo strategico della comunicazione quale componente strutturale delle organizzazioni e dei processi di business. Tuttavia, questo tema è affrontato nell'articolo dal punto di vista della comunicazione come leva a supporto del marketing e non della comunicazione strategica.

\section{Obiettivi dello studio e metodologia della ricerca}

Il percorso metodologico che ha avuto inizio con la literature review è servito ad esaminare i contributi più recenti in materia di marketing e comunicazione nelle PMI e ad individuare le peculiarità specifiche di questa tipologia di impresa. Quasi tutti gli Autori sono concordi col considerare le PMI poco propense ad adottare specifiche attività di marketing e comunicazione essendo, dai loro punti di vista, caratterizzate da una gestione del marketing mix quasi inesistente. Benché le PMI siano una realtà solida e dinamica e gestiscano la maggior parte del business 
nazionale, ciò che si evidenzia dalla review della letteratura è la loro scarsa capacità a proiettarsi in un sistema competitivo evoluto, che contempli, tra le altre cose, l'investimento in attività pianificate di marketing e comunicazione. Partendo da tali considerazioni iniziali, l'articolo intende: 1) verificare attraverso una ricerca sul campo, se tali evidenze siano ancora valide e rispondere a tale interrogativo: "Nell'attuale contesto competitivo le PMI continuano ad essere diffidenti nei confronti del marketing e della comunicazione?"; 2) contribuire ad arricchire la letteratura in materia con una ricerca cross-country Italia-Inghilterra.

Al fine di rispondere all'interrogativo di ricerca e perseguire gli obiettivi enunciati è stata progettata la ricerca empirica suddividendola in due fasi. La prima quantitativa, volta a raccogliere le informazioni generali sul comportamento delle PMI nei riguardi del marketing e della comunicazione. Una seconda fase qualitativa, tesa ad approfondire le tematiche maggiormente innovative o significative emerse nella fase quantitativa. Infine, attraverso la cluster analysis si è provveduto ad individuare gruppi di imprese con caratteristiche simili, attraverso la profilazione di tre atteggiamenti tipo.

È stata progettata una web-survey per raccogliere le informazioni attraverso un questionario strutturato su scale single e multi-item. La web survey è stata creata servendosi del sito internet hosting: www.surveymonkey.com che ha permesso di creare e inserire il questionario direttamente online. Attraverso un software apposito (pop-up survey), la survey ha raccolto i dati delle PMI che visitando la piattaforma, hanno compilato il questionario. La survey è stata strutturata in tre parti in modo da consentirci di indagare diverse aree conoscitive: una parte generale volta a identificare e classificare l'impresa rispondente (anno di fondazione, fatturato, numero di dipendenti); una parte relativa alle attività di marketing adottate ed infine una parte relativa alle attività di comunicazione implementate. Le questioni di fondo indagate riguardano: l'uso delle tecniche di marketing e comunicazione; l'uso delle strutture coinvolte; il ricorso a collaborazioni esterne; lo sviluppo futuro previsto in termini di investimenti. L'indagine web-based è stata scelta per consentire alle imprese del campione di indicare le loro risposte in un ambiente "familiare", di facile ed immediato utilizzo, che non comportasse un eccessivo dispendio di tempo e che perciò invogliasse maggiormente a rispondere al questionario. Nonostante lo sforzo profuso è stata, comunque, riscontrata una generale avversione delle PMI a fornire risposte e informazioni a riguardo.

La ricerca è stata perciò, inficiata da questa difficoltà di base, ed è stata, dunque, condotta su un campione di convenienza, frutto di un processo di campionamento non probabilistico, che vede coinvolte 100 PMI (74 italiane e 26 inglesi), nel periodo febbraio-novembre 2011. Purtroppo a causa della difficoltà di reperire risposte da parte delle PMI si è optato per la scelta di un campionamento non probabilistico (limite della ricerca). Il lavoro non presenta, almeno nella sua parte dedicata alle imprese inglesi, un campione numericamente significativo. 


\section{I due campioni di imprese}

L'individuazione degli elementi strutturali (dimensione, fatturato, mercati di riferimento) è stato il punto di partenza per delineare le caratteristiche delle imprese sottoposte a comparazione. I due campioni di imprese presentano al loro interno una forte eterogeneità (Tab. 3). Nel campione italiano, i settori che registrano una frequenza maggiore sono i seguenti: metalmeccanico (20), servizi (17) e alimentare (15). Gli altri settori (manifatturiero, ICT, tessile e chimico) registrano una minore frequenza. Nel campione inglese, il settore che registra la frequenza maggiore è quello dei servizi (16). I settori che seguono, in termini di frequenza, sono quello alimentare (6), ed infine i settori manifatturiero e quello dell'ICT. La quasi totalità delle imprese dei due campioni opera nel B-to-B. Infatti, solo il $21,1 \%$ delle imprese italiane opera nel B-to-C, mentre questo dato è totalmente assente nel campione inglese. Le imprese italiane coprono in maniera eterogenea le vari classi di fatturato, mentre la maggior parte delle imprese inglesi del campione $(64,7 \%)$ ha un fatturato basso (fino a 2 milioni). Gran parte delle imprese italiane $(43,2 \%)$ ha una classe di dipendenti che va da 51 a 250. Al contrario, quelle inglesi coprono la classe più esigua di dipendenti compresa tra 0 fino a 10 dipendenti $(53,8 \%)$.

Tab. 3: Caratteristiche strutturali delle PMI dei due campioni

\begin{tabular}{|c|c|c|}
\hline Caratteristiche & Imprese italiane & Imprese inglesi \\
\hline $\begin{array}{l}\text { SETTORI } \\
\text { metalmeccanico } \\
\text { alimentare } \\
\text { servizi } \\
\text { manifatturiero } \\
\text { ICT } \\
\text { tessile } \\
\text { chimico } \\
\end{array}$ & $\begin{array}{c}27,0 \% \\
21,1 \% \\
22,9 \% \\
9,5 \% \\
6,5 \% \\
6,5 \% \\
6.5 \% \\
\end{array}$ & $\begin{array}{c}--- \\
23,3 \% \\
61,5 \% \\
7,6 \% \\
7,6 \% \\
--- \\
--- \\
\end{array}$ \\
\hline $\begin{array}{l}\text { TARGET } \\
B \text { to } B \\
B \text { to } C \\
\end{array}$ & $\begin{array}{l}78,9 \% \\
21,1 \% \\
\end{array}$ & $\begin{array}{c}100 \% \\
---\end{array}$ \\
\hline $\begin{array}{l}\text { FATTURATO }(€) \\
\text { fino a } 2 \text { milioni } \\
\text { da } 2 \text { a } 10 \text { milioni } \\
\text { da } 10 \text { a } 50 \text { milioni } \\
\text { oltre i } 50 \text { milioni }\end{array}$ & $\begin{array}{l}17,5 \% \\
23,8 \% \\
19,0 \% \\
39,7 \% \\
\end{array}$ & $\begin{array}{c}64,7 \% \\
23,5 \% \\
11,8 \% \\
--- \\
\end{array}$ \\
\hline $\begin{array}{l}\text { DIPENDENTI } \\
\text { fino a } 10 \\
\text { da } 2 \text { fino a } 50 \\
\text { da } 51 \text { fino a } 250 \\
\text { superiore a } 250\end{array}$ & $\begin{array}{c}4,1 \% \\
35,1 \% \\
43,2 \% \\
17,6 \% \\
\end{array}$ & $\begin{array}{c}53,8 \% \\
23,1 \% \\
23,1 \% \\
--- \\
\end{array}$ \\
\hline
\end{tabular}

Fonte: ns. elaborazione 


\section{I risultati}

L'analisi empirica condotta ha avuto come obiettivo quello di studiare il comportamento, le modalità e le strategie marketing e comunicazione delle piccole e medie imprese, al fine di tracciare un quadro di sintesi della situazione attuale in Italia e in Inghilterra. Dall'indagine svolta risulta che le PMI manifestano più o meno consapevolmente la necessità di adottare specifiche attività di marketing e comunicazione, in vista del perseguimento di determinati obiettivi, quali: l'aumento delle vendite e il miglioramento della quota di mercato; il consolidamento dell'immagine aziendale; la soddisfazione dei clienti e la promozione della fedeltà, l'opportunità di entrare in nuovi mercati e lo sviluppo della reputazione (Tab. 4). Dalla tabella proposta, è facile dedurre che le imprese italiane prestano molta attenzione agli obiettivi concernenti l'aumento delle vendite e il miglioramento della quota di mercato. Allo stesso modo le imprese inglesi prestano attenzione a questi obiettivi anche se si evidenzia in questo caso un' attenzione alla reputazione, quale intangible asset per garantire il raggiungimento di un vantaggio competitivo duraturo. Questo obiettivo risulta invece, secondario nelle imprese italiane le quali sembrano prestare minore attenzione alla costruzione di una forte corporate reputation.

Tab. 4: Gli obiettivi delle imprese studiate

\begin{tabular}{|l|c|c|}
\hline \multicolumn{1}{|c|}{ Obiettivi } & $\begin{array}{c}\text { Imprese } \\
\text { Italiane }\end{array}$ & $\begin{array}{c}\text { Imprese } \\
\text { Inglesi }\end{array}$ \\
\hline Aumentare le vendite e migliorare la quota di mercato & $\mathbf{7 7 . 0 \%}$ & $\mathbf{9 2 , 3 \%}$ \\
\hline Migliorare l'immagine aziendale & $\mathbf{6 0 , 8 \%}$ & $\mathbf{5 0 , 0 \%}$ \\
\hline Soddisfare i clienti e promuovere la fedeltà & $\mathbf{5 4 , 1 \%}$ & $30,8 \%$ \\
\hline Valutare l'opportunità di entrare in nuovi mercati & $\mathbf{4 5 , 9 \%}$ & $\mathbf{2 3 , 1 \%}$ \\
\hline Sviluppare la reputazione & $\mathbf{2 7 . 0 \%}$ & $\mathbf{5 7 , 7 \%}$ \\
\hline
\end{tabular}

Fonte: ns. elaborazione

Tab. 5: Le attività di marketing e comunicazione adottate nelle imprese italiane

\begin{tabular}{|l|c|c|c|c|}
\hline $\begin{array}{c}\text { Attività di marketing e } \\
\text { comunicazione }\end{array}$ & non svolta & saltuaria & frequente & continuativa \\
\hline segmentazione & $25,7 \%$ & $28,4 \%$ & $\mathbf{3 1 , 1} \%$ & $\mathbf{1 4 , 9 \%}$ \\
\hline targeting & 17,8 & $\mathbf{3 4 , 2} \%$ & $\mathbf{3 4 , 2} \%$ & $\mathbf{1 3 , 7 \%}$ \\
\hline posizionamento & $18,1 \%$ & $19,4 \%$ & $\mathbf{4 0 , 3} \%$ & $\mathbf{2 2 , 2} \%$ \\
\hline sviluppo nuovi prodotti & $9,6 \%$ & $17,8 \%$ & $35,6 \%$ & $\mathbf{3 7 , 0} \%$ \\
\hline decisioni di packaging & $\mathbf{3 3 , 8} \%$ & $26,8 \%$ & $22,5 \%$ & $\mathbf{1 6 , 9 \%}$ \\
\hline decisioni di prezzo & $23,0 \%$ & $14,9 \%$ & $29,7 \%$ & $\mathbf{3 2 , 4 \%}$ \\
\hline decisioni di distribuzione & $\mathbf{3 5 , 2} \%$ & $16,9 \%$ & $\mathbf{3 2 , 4 \%}$ & $\mathbf{1 5 , 5 \%}$ \\
\hline decisioni di comunicazione & $5,5 \%$ & $19,2 \%$ & $\mathbf{3 9 , 7} \%$ & $\mathbf{3 5 , 6 \%}$ \\
\hline operazioni di branding & $16,9 \%$ & $\mathbf{3 3 , 8} \%$ & $32,4 \%$ & $\mathbf{1 6 , 9 \%}$ \\
\hline CRM & $21,1 \%$ & $\mathbf{3 2 , 4} \%$ & $26,8 \%$ & $\mathbf{1 9 , 7 \%}$ \\
\hline ricerche di mercato & $27,1 \%$ & $\mathbf{3 8 , 6 \%}$ & $24,3 \%$ & $\mathbf{1 0 , 0 \%}$ \\
\hline trade marketing & $29,2 \%$ & $\mathbf{3 0 , 6} \%$ & $25,0 \%$ & $\mathbf{1 5 , 3 \%}$ \\
\hline
\end{tabular}

Fonte: ns. elaborazione 
Allo scopo di comprendere il livello di avanzamento del marketing concept all'interno delle imprese studiate, è stato chiesto di precisare se una serie di attività venissero svolte o meno e con quale frequenza.

Come si può evincere dalla tabella 5 , le attività svolte in maniera costante e continuativa nelle imprese italiane del campione sono: le decisioni di sviluppo nuovi prodotti; le decisioni di prezzo; le decisioni di comunicazione. Le imprese inglesi del campione svolgono in maniera continuativa decisioni di comunicazione; attività di CRM; decisioni di prezzo (Tab. 6).

Tab. 6: Le attività di marketing e comunicazione adottate nelle imprese inglesi

\begin{tabular}{|l|r|r|r|r|}
\hline \multicolumn{1}{|c|}{$\begin{array}{c}\text { Attività di marketing e } \\
\text { comunicazione }\end{array}$} & non svolta & saltuaria & frequente & continuativa \\
\hline segmentazione & $32,0 \%$ & $\mathbf{4 0 , 0} \%$ & $16,0 \%$ & $\mathbf{1 2 , 0} \%$ \\
\hline targeting & $11,5 \%$ & $30,8 \%$ & $\mathbf{3 8 , 5} \%$ & $\mathbf{1 9 , 2} \%$ \\
\hline posizionamento & $16,0 \%$ & $\mathbf{3 6 , 0} \%$ & $32,0 \%$ & $\mathbf{1 6 , 0} \%$ \\
\hline sviluppo nuovi prodotti & $26,9 \%$ & $23,1 \%$ & $30,8 \%$ & $\mathbf{1 9 , 2} \%$ \\
\hline decisioni di packaging & $\mathbf{4 4 , 0} \%$ & $32,0 \%$ & $16,0 \%$ & $\mathbf{8 , 0} \%$ \\
\hline decisioni di prezzo & $23,1 \%$ & $26,9 \%$ & $19,2 \%$ & $\mathbf{3 0 , 8} \%$ \\
\hline decisioni di distribuzione & $\mathbf{4 2 , 3} \%$ & $26,9 \%$ & $11,5 \%$ & $\mathbf{1 9 , 2} \%$ \\
\hline decisioni di comunicazione & $11,5 \%$ & $\mathbf{3 8 , 5} \%$ & $15,4 \%$ & $\mathbf{3 4 , 6 \%}$ \\
\hline operazioni di branding & $15,4 \%$ & $\mathbf{5 0 , 0} \%$ & $15,4 \%$ & $\mathbf{1 9 , 2} \%$ \\
\hline CRM & $15,4 \%$ & $11,5 \%$ & $\mathbf{3 8 , 5} \%$ & $\mathbf{3 4 , 6 \%}$ \\
\hline ricerche di mercato & $11,5 \%$ & $\mathbf{4 6 , 2} \%$ & $30,8 \%$ & $\mathbf{1 1 , 5 \%}$ \\
\hline trade marketing & $29,2 \%$ & $\mathbf{4 1 , 7} \%$ & $20,8 \%$ & $\mathbf{8 , 3} \%$ \\
\hline
\end{tabular}

Fonte: ns. elaborazione

L'analisi delle attività di comunicazione ricalca in gran parte la sequenza illustrata nell'esame delle attività di marketing. Innanzitutto, è stato richiesto di precisare le forme e i mezzi di comunicazione utilizzati e le modalità di svolgimento degli stessi (non svolta, svolta in modo saltuario, frequente o continuativo). Le imprese italiane del campione utilizzano in maniera continuativa: il passaparola $(30,4 \%)$; la promozione delle vendite $(26,8 \%)$; la vendita personale $(24,3 \%)$. Le altre forme di comunicazione, come le relazioni pubbliche offline $(30,3 \%)$, la pubblicità (advertising) (29,3\%), l'e-advertising (pubblicità web, banner pubblicitari, social network) (28\%) e le sponsorizzazioni (20,3\%), sono utilizzate in maniera frequente (Tab. 7).

Le piccole e medie imprese inglesi utilizzano in maniera continuativa (Tab. 8): il passaparola $(52,0 \%)$; la vendita personale $(44,0 \%)$; e le relazioni pubbliche $(24 \%)$. Sono invece utilizzate in maniera saltuaria le altre forme di comunicazione: la pubblicità (advertising) (38,5\%); le sponsorizzazioni (46,2\%); il direct marketing (telemarketing, mailing, etc.) (46,2\%).

Le piccole e medie imprese inglesi utilizzano in maniera continuativa (tab. 7): il passaparola $(52,0 \%)$; la vendita personale $(44,0 \%)$; e le relazioni pubbliche $(24 \%)$. Sono invece utilizzate in maniera saltuaria le altre forme di comunicazione: la pubblicità (advertising) (38,5\%); le sponsorizzazioni (46,2\%); il direct marketing (telemarketing, mailing, etc.) $(46,2 \%)$. 
Le PMI italiane ed inglesi utilizzano in maniera continuativa internet e i new media con percentuali molto alte.

Tab. 7: Le forme e i mezzi di comunicazione adottati nelle imprese italiane

\begin{tabular}{|c|c|c|c|c|}
\hline $\begin{array}{l}\text { Forme e mezzi di } \\
\text { comunicazione }\end{array}$ & $\begin{array}{c}\text { non } \\
\text { utilizza }\end{array}$ & saltuaria & frequente & continuativa \\
\hline pubblicità & $6,7 \%$ & $45,3 \%$ & $29,3 \%$ & $18,7 \%$ \\
\hline e-advertising & $18,7 \%$ & $33,3 \%$ & $28,0 \%$ & $20,0 \%$ \\
\hline sponsorizzazioni & $14,9 \%$ & $54,1 \%$ & $20,3 \%$ & $10,8 \%$ \\
\hline relazioni pubbliche online & $31,9 \%$ & $41,7 \%$ & $12,5 \%$ & $13,9 \%$ \\
\hline relazioni pubbliche offline & $15,8 \%$ & $28,9 \%$ & $30,3 \%$ & $25,0 \%$ \\
\hline direct marketing & $39,4 \%$ & $22,5 \%$ & $21,1 \%$ & $16,9 \%$ \\
\hline promozione vendite & $29,6 \%$ & $19,7 \%$ & $23,9 \%$ & $26,8 \%$ \\
\hline vendita personale & $44,6 \%$ & $20,3 \%$ & $10,8 \%$ & $24,3 \%$ \\
\hline passaparola & $30,4 \%$ & $21,7 \%$ & $17,4 \%$ & $30,4 \%$ \\
\hline stampa e redazionali & $6,6 \%$ & $43,4 \%$ & $22,4 \%$ & $27,6 \%$ \\
\hline cartellonistica e affissioni & $58,1 \%$ & $23,0 \%$ & $14,9 \%$ & $4,1 \%$ \\
\hline eventi & $3,9 \%$ & $36,4 \%$ & $40,3 \%$ & $19,5 \%$ \\
\hline televisione & $68,0 \%$ & $24,0 \%$ & $5,3 \%$ & $2,7 \%$ \\
\hline radio & $71,2 \%$ & $26,0 \%$ & $2,7 \%$ & $0,0 \%$ \\
\hline mezzi pubblici & $70,8 \%$ & $22,2 \%$ & $6,9 \%$ & $0,0 \%$ \\
\hline internet/new media & $9,5 \%$ & $23,0 \%$ & $23,0 \%$ & $44,6 \%$ \\
\hline
\end{tabular}

Fonte: ns. elaborazione

Tab. 8: Le forme e i mezzi di comunicazione adottati nelle imprese inglesi

\begin{tabular}{|c|c|c|c|c|}
\hline $\begin{array}{l}\text { Forme e mezzi di } \\
\text { comunicazione }\end{array}$ & $\begin{array}{c}\text { non } \\
\text { utilizza }\end{array}$ & saltuaria & frequente & continuativa \\
\hline pubblicità (advertising) & $34,6 \%$ & $38,5 \%$ & $11,5 \%$ & $15,4 \%$ \\
\hline e-advertising & $46,2 \%$ & $19,2 \%$ & $19,2 \%$ & $15,4 \%$ \\
\hline sponsorizzazioni & $42,3 \%$ & $46,2 \%$ & $7,7 \%$ & $3,8 \%$ \\
\hline relazioni pubbliche on line & $32,0 \%$ & $24,0 \%$ & $20,0 \%$ & $24,0 \%$ \\
\hline relazioni pubbliche off line & $32,0 \%$ & $28,0 \%$ & $20,0 \%$ & $20,0 \%$ \\
\hline direct marketing & $34,6 \%$ & $46,2 \%$ & $15,4 \%$ & $3,8 \%$ \\
\hline promozione vendite & $42,3 \%$ & $30,8 \%$ & $19,2 \%$ & $7,7 \%$ \\
\hline vendita personale & $24,0 \%$ & $12,0 \%$ & $20,0 \%$ & $44,0 \%$ \\
\hline passaparola & $8,0 \%$ & $12,0 \%$ & $28,0 \%$ & $52,0 \%$ \\
\hline stampa e redazionali & $6,6 \%$ & $43,4 \%$ & $22,4 \%$ & $27,6 \%$ \\
\hline cartellonistica e affissioni & $58,1 \%$ & $23,0 \%$ & $14,9 \%$ & $4,1 \%$ \\
\hline eventi & $3,9 \%$ & $36,4 \%$ & $40,3 \%$ & $19,5 \%$ \\
\hline televisione & $68,0 \%$ & $24,0 \%$ & $5,3 \%$ & $2,7 \%$ \\
\hline radio & $71,2 \%$ & $26,0 \%$ & $2,7 \%$ & $0,0 \%$ \\
\hline mezzi pubblici & $70,8 \%$ & $22,2 \%$ & $6,9 \%$ & $0,0 \%$ \\
\hline internet/new media & $9,5 \%$ & $23,0 \%$ & $23,0 \%$ & $44,6 \%$ \\
\hline
\end{tabular}

Fonte: ns. elaborazione

Nessuna tra le imprese dei due campioni prevede di diminuire gli investimenti di marketing e comunicazione in termini di risorse umane e finanziarie. All'opposto, 
molte attività vengono segnalate in attesa di maggiori investimenti rispetto a quelli precedentemente effettuati. Con l'obiettivo di tracciare lo stato attuale e futuro dei futuri investimenti, sono state individuate le attività destinate a subire un aumento, una diminuzione o un mantenimento dell'investimento, nei due campioni studiati (Tab. 9).

Tab. 9: Gli investimenti in marketing e comunicazione nei due campioni di imprese

\begin{tabular}{|l|r|r|}
\hline \multicolumn{1}{|c|}{ Investimenti in marketing e comunicazione } & Imprese Italiane & Imprese inglesi \\
\hline più o meno come prima & $\mathbf{4 5 , 5} \%$ & $\mathbf{3 0 , 8} \%$ \\
\hline più di prima & $\mathbf{3 7 , 7} \%$ & $\mathbf{3 8 , 5} \%$ \\
\hline $\begin{array}{c}\text { Attività che subiranno un aumento } \\
\text { di investimenti }\end{array}$ & Imprese Italiane & Imprese inglesi \\
\hline ricerche di mercato & $\mathbf{2 0 , 6} \%$ & $\mathbf{2 9 , 4} \%$ \\
\hline decisioni di packaging & $\mathbf{2 1 , 9} \%$ & $11,7 \%$ \\
\hline CRM & $8,2 \%$ & $\mathbf{1 7 , 6} \%$ \\
\hline pubblicità & $\mathbf{3 0 , 1} \%$ & $11,1 \%$ \\
\hline relazioni pubbliche & $12,3 \%$ & $\mathbf{2 9 , 4} \%$ \\
\hline internet/new media & $\mathbf{3 0 , 8} \%$ & $\mathbf{2 9 , 4} \%$ \\
\hline eventi & $\mathbf{3 1 , 7} \%$ & $17,6 \%$ \\
\hline
\end{tabular}

Fonte: ns. elaborazione

Dall'analisi delle risposte si segnala una predisposizione all'aumento degli investimenti molto forte nei riguardi delle nuove tecnologie dell'informazione connesse ad internet. Risulta che i due campioni di imprese, intendono prestare in futuro maggiore attenzione allo sviluppo del prodotto. Il $61,1 \%$ delle imprese italiane del campione e il $41,7 \%$ delle imprese inglesi confermano che il prossimo investimento in tale attività registrerà un progressivo aumento. Le altre attività che registrano la propensione ad un aumento degli investimenti sono il CRM, la customer care, l'organizzazione degli eventi, anche se in questi casi le percentuali sono più basse.

Per cercare di ricostruire l'organizzazione della funzione marketing/ comunicazione delle piccole e medie imprese analizzate, si è proceduto in due fasi. Si è partiti con l'identificazione del numero degli impiegati coinvolti nella gestione della funzione all'interno dell'organigramma aziendale. Successivamente si è proceduto ad individuare i ruoli ricoperti nell'unità organizzativa dedicata alla gestione del marketing e della comunicazione. Dall'analisi dei risultati conseguiti, è emerso che nelle imprese italiane e in quelle inglesi si adoperano mediamente tra i 2 e 4 addetti nella gestione della comunicazione e del marketing. Nella fase successiva si è proceduto ad individuare $\mathrm{i}$ ruoli ricoperti più di frequente nell'unità organizzativa dedicata (Tab. 10).

Le figure manageriali e quelle di direzione sono quelle più frequenti nei due campioni. Si rileva la presenza in quasi tutti gli organigrammi aziendali del direttore marketing. Le figure operative sono altresì importanti e hanno compiti che vanno dall'organizzazione degli eventi, alle sponsorizzazioni. Esse fungono da assistant nei riguardi del direttore o dei manager della relativa funzione. Sono inoltre presenti in molte delle imprese studiate operatori telemarketing o web marketing specialists. 
Tab. 10: Il ricorso ai consulenti esterni nei due campioni

\begin{tabular}{|l|c|c|}
\hline $\begin{array}{l}\text { Ruoli ricoperti nell'unità organizzativa dedicata alla gestione } \\
\text { del marketing e della comunicazione? }\end{array}$ & $\begin{array}{c}\text { Frequenza } \\
\text { Imprese } \\
\text { italiane }\end{array}$ & $\begin{array}{c}\text { Frequenza } \\
\text { Imprese } \\
\text { Inglesi }\end{array}$ \\
\hline Direttore marketing e/o marketing manager & $\mathbf{6 5}$ & $\mathbf{1 8}$ \\
\hline Ufficio stampa, PR e organizzazione eventi & $\mathbf{2 5}$ & $\mathbf{1 0}$ \\
\hline Addetto al marketing/comunicazione & 13 & 8 \\
\hline Ricerche di mercato e CRM & 11 & 5 \\
\hline Direttore commerciale/Resp. vendite & 9 & 4 \\
\hline Operatore telemarketing & 7 & 3 \\
\hline Web specialist /web marketing & 7 & 2 \\
\hline
\end{tabular}

Fonte: ns. elaborazione

\section{La cluster analysis}

A conclusione del lavoro è stata effettuata una cluster analysis con l'obiettivo di sintetizzare i risultati. La cluster analysis è, infatti, una tecnica esplorativa e descrittiva, che serve a ridurre la complessità delle unità di indagine. Per effettuare tale analisi è stato utilizzato un software metodologico "SPAD" che attraverso l'analisi delle corrispondenze multiple ha consentito di ridurre il complesso spazio delle variabili a pochi fattori principali in grado di spiegare la maggior parte della variabilità della matrice dei dati. Sono stati individuati tre gruppi (cluster) di imprese con caratteristiche simili intra gruppo e dissimili inter gruppo:

- imprese proattive;

- imprese moderate;

- imprese scettiche.

Ognuno dei tre cluster presenta al proprio interno peculiarità e caratteristiche specifiche. Allo scopo di individuare le peculiarità di tali cluster, sono state create diverse tavole di contingenza che hanno restituito diverse informazioni, utili a caratterizzare i tre profili (settore di appartenenza, fatturato, numero di dipendenti, target). Il profilo dell'impresa proattiva (Tab. 11) sintetizza le peculiarità strutturali di un'impresa di media dimensione, molto propensa all'adozione di specifiche attività di marketing e comunicazione. È un'impresa che mostra un'alta propensione agli investimenti in questo ambito. Le imprese proattive convergono nel settore dell'ICT (75\%), seguito da quelli chimico e alimentare (entrambi con il 60\%).

Tab. 11: Profilo dell'impresa proattiva

\begin{tabular}{|l|l|}
\hline \multicolumn{1}{|c|}{ Caratteristiche } & \multicolumn{1}{c|}{ Dati } \\
\hline Profilo aziendale & Media impresa (fatturato da 10 milioni a 50 milioni - $\mathrm{n}^{\circ}$ dipendenti: da 50 a 250) \\
\hline Settore & ICT \\
\hline Nazionalità & Italiana \\
\hline Atteggiamento & Atteggiamento verso il marketing: caldo (86\%) \\
\hline Investimenti & Alta propensione agli investimenti in marketing e comunicazione (56\%) \\
\hline Attività & Alta intensità d'uso delle attività di marketing e comunicazione \\
\hline
\end{tabular}

Fonte: ns. elaborazione 
Il profilo dell'impresa "moderata" (Tab. 12) riguarda un'impresa ancora di media dimensione che mostra al contrario una minore propensione agli investimenti in marketing e comunicazione. I settori in cui prevale questa tipologia di imprese sono quello tessile e chimico.

Tab. 12: Profilo dell'impresa reattiva

\begin{tabular}{|l|l|}
\hline Caratteristiche & \multicolumn{1}{c|}{ Dati } \\
\hline Profilo aziendale & $\begin{array}{l}\text { Media impresa (fatturato da 10 milioni a } 50 \text { milioni - } \mathrm{n}^{\circ} \text { dipendenti: da } 50 \text { a } \\
250)\end{array}$ \\
\hline Settore & Tessile e chimico \\
\hline Nazionalità & Italiana ed inglese \\
\hline Atteggiamento & Atteggiamento verso il marketing: caldo \\
\hline Investimenti & Moderata propensione agli investimenti in marketing e comunicazione \\
\hline Attività & Bassa intensità d'uso delle attività di marketing e comunicazione \\
\hline
\end{tabular}

Fonte: ns. elaborazione

Il profilo dell'impresa "scettica" (Tab. 13) delinea un'impresa, che, in linea con il suo limite dimensionale ( si tratta di una micro impresa, con un fatturato fino a due milioni e un $\mathrm{n}^{\circ}$ dipendenti fino a 10), fatica a intraprendere un percorso che consenta di intraprendere specifiche attività di marketing e comunicazione. Si tratta di imprese che mostrano un atteggiamento negativo nei riguardi del marketing con una bassa propensione agli investimenti in questo ambito. I settori in cui convergono queste imprese sono quello dei servizi, metalmeccanico e manifatturiero.

Tab. 13: Profilo dellimpresa scettica

\begin{tabular}{|l|l|}
\hline Caratteristiche & \multicolumn{1}{c|}{ Dati } \\
\hline Profilo aziendale & Piccola impresa (fatturato: fino a due milioni - $\mathrm{n}^{\circ}$ dipendenti: fino a 10) \\
\hline Settore & Servizi \\
\hline Nazionalità & Inglese \\
\hline Atteggiamento & Atteggiamento verso il marketing: freddo \\
\hline Investimenti & Bassa propensione agli investimenti in marketing e comunicazione \\
\hline Attività & Bassa intensità d'uso delle attività di marketing e comunicazione \\
\hline
\end{tabular}

Fonte: ns. elaborazione

I profili delineati sono serviti a fornire un quadro di sintesi delle imprese studiate che, a seconda delle proprie caratteristiche e peculiarità, esprimono specifici comportamenti di marketing e comunicazione. L'evidenza più rilevante che emerge dall'analisi è che le differenze all'interno dei cluster sono legate a fattori dimensionali o industry specific piuttosto che di localizzazione geografica. Non si segnalano, infatti, significative differenze tra le imprese italiane ed inglesi. Piuttosto, le imprese più grandi (medie imprese) sia di nazionalità italiana che inglese generalmente tendono ad utilizzare un spettro di attività di marketing e comunicazione più ampio e completo. Al contrario, le imprese più piccole (micro imprese), non avendo a disposizione risorse informative ed umane sufficienti, non 
dedicano al marketing e alla comunicazione sufficiente attenzione in termini di risorse, politiche e strumenti, relegandole ad attività sporadiche ed improvvisate, con performance poco soddisfacenti ai fini della costruzione e del sostegno del proprio posizionamento competitivo. Inoltre, le differenze tra i diversi cluster sono altresì legate ai settori di appartenenza delle imprese dei due campioni, come si evince dalla lettura dei tre profili delineati.

\section{Le interviste strutturate}

A sostegno dei risultati ottenuti, è stata prevista nel corso di questa ricerca, una fase di analisi qualitativa utile a irrobustire i risultati scaturiti dall'indagine quantitativa. La ricerca si è avvalsa di interviste strutturate. Sono stati coinvolti tutti i manager della funzione marketing/comunicazione delle 100 imprese (74 italiane e 26 inglesi) coinvolte prima fase dell'indagine nel periodo Novembre 2011-Febbraio 2012. In questo caso, le risposte non sono state numericamente significative e si è riscontrata la sola partecipazione delle imprese italiane. Le interviste sono state formulate con l'intento di indagare l'approccio delle imprese nei confronti del marketing e della comunicazione, le maggiori difficoltà e le opportunità di sviluppo futuro.

Dalla lettura dei risultati emerge uno scenario lacunoso e pessimistico che si allontana da quello positivo e ottimistico tracciato dall'indagine quantitativa. Infatti, la maggior parte dei manager intervistati ritiene che l'attività di marketing e comunicazione venga svolta solo di riflesso nelle PMI, perché mancano i presupposti e le condizioni per poter adottare un approccio proattivo. Secondo alcuni, "il marketing è gestito in maniera improvvisata, raramente da strutture competenti e spesso da dilettanti di livello mediocre".

Inoltre, gli stessi manager ritengono che le piccole e medie imprese si interfaccino al marketing soltanto in alcuni casi. La scelta di adottare o meno specifiche attività è dettata dal settore, dal contesto di appartenenza e dalla situazione in cui versa l'azienda. In molti casi, mancano strutture e uffici predisposti alla gestione delle attività di marketing e comunicazione nonché gli specialisti e le figure professionali competenti in questo ambito. La carenza di personale ad hoc e la mancanza di strutture competenti è un tema molto controverso, assai dibattuto anche in letteratura, che si conferma uno dei motivi su cui maggiormente indagare per comprendere perché le imprese di più piccola dimensione non riescono ad approcciare il marketing nel modo giusto. Un'altra grossa debolezza rilevata dai manager intervistati è la mancata utilizzazione in molte PMI di modalità di analisi e pianificazione strategica. Tuttavia, gli stessi sono concordi nel ritenere fondamentale tale attività, che permette alle imprese di piccole e medie dimensioni di stabilire gli obiettivi da perseguire e le risorse da utilizzare". Inoltre, è convinzione consolidata che "le piccole imprese si reggono soprattutto sulle esperienze quotidiane di chi le governa". 
Infine, gli stessi manager intravedono in questo scenario un cambiamento nel modo di fare marketing e comunicazione, già in atto per alcuni aspetti. Infatti, è opinione consolidata che "i clienti vorranno percepire cose diverse e la comunicazione ai consumatori giocherà un ruolo fondamentale".

\section{Sommario e conclusioni}

Nell'attuale scenario competitivo, la comunicazione e il marketing sono al centro dell'attenzione delle piccole e medie imprese che hanno riconosciuto l'idea che il know-how comunicativo deve costituire una competenza diffusa in tutta l'organizzazione. Alla luce dei risultati scaturiti dalla ricerca empirica, è evidente che a differenza di quanto rilevato in letteratura, le PMI iniziano a riconoscere l'importanza del marketing e della comunicazione ricorrendo ad una massiccia utilizzazione delle attività ad esse connesse. Gli investimenti in marketing e comunicazione vengono, infatti, considerati come un fattore determinante nel creare e mantenere una differenziazione ed un vantaggio d'immagine presso la clientela. Il $61,1 \%$ delle imprese italiane e il $41,7 \%$ di quelle inglesi confermano un maggiore investimento in tale attività nei prossimi anni.

In disaccordo con quanto ritenuto in passato (relativamente ai contributi analizzati), nella maggiore parte delle imprese intervistate, le attività di marketing $\mathrm{e}$ comunicazione risultano essere entrate nelle routine aziendali, con una crescente predisposizione all'utilizzo dei new media e di internet. Infatti, sia le imprese italiane che quelle inglesi adottano in maniera continuativa internet e i new media con una percentuale del $44.6 \%$.

La presenza di un crescente numero di addetti nella Direzione dedicata alla gestione del marketing e della comunicazione in quasi tutti gli organigramma aziendali rappresenta un segnale positivo sull'evoluzione del marketing nelle aziende analizzate. Infatti, dalla lettura dei risultati conseguiti, è emerso che nelle imprese italiane e in quelle inglesi si adoperano mediamente tra i 2 e 4 addetti nella gestione della comunicazione e del marketing.

Il quadro che emerge dall'indagine è interessante: il marketing e la comunicazione permeano l'attività della gran parte delle imprese, sempre più attente a seguirne l'evoluzione sia in termini di strategie sia di strumenti operativi.

Tuttavia, la scelta di adottare un approccio proattivo nei confronti del marketing e della comunicazione è condizionato da alcune ragioni intrinseche all'impresa come il settore di appartenenza, la dimensione, ecc. Al contrario, la localizzazione geografica ha un peso minore come evidenziato dalla comparazione cross-country Italia-Inghilterra. Infatti, ciò che si evince dalla lettura dei risultati è che la dimensione delle imprese (intesa in relazione al numero di addetti e fatturato) influenza la tipologia di approccio al marketing e alla comunicazione. Pertanto, le imprese di dimensione maggiore (medie imprese), confermano l'utilizzo frequente o continuativo delle attività di marketing e comunicazione a differenza di quelle di più 
piccola dimensione (micro e piccole imprese) che sono costrette ad un uso sporadico di queste attività.

Un interessante segnale emerge dalla lettura dei risultati scaturiti dalla cluster analysis. Raggruppando le imprese analizzate sulla base del tipo di offerta e dei mercati di riferimento si può notare che il settore in cui si registra una maggiore frequenza di imprese scettiche, è quello dei servizi. Si tratta di imprese che scontano qualche ritardo e in cui non si adoperano strumenti di marketing e comunicazione, come confermato anche dagli studi analizzati in letteratura (Hätönen e Ruokonen, 2010). A conclusione del lavoro, le principali tendenze emerse sono le seguenti:

- emerge una significativa crescita di importanza dei nuovi mezzi di comunicazione: internet e i new media. La loro diffusione ed uso sono in forte aumento come dimostrano i dati sui crescenti ulteriori investimenti nel prossimo futuro, all'interno di un panorama dove tutti gli altri strumenti sono in forte stabilità (circa il 30\% delle imprese aumenterà gli investimenti in tale ambito);

- si nota, in generale, una costante crescita della cultura di marketing e di comunicazione delle organizzazioni;

- è evidente che il comportamento delle imprese nei confronti del marketing e della comunicazione dipende dalle caratteristiche intrinseche delle PMI (settore di appartenenza, dimensione, target);

- il paradigma della dimensione influenza la tipologia di approccio al marketing e alla comunicazione.

I risvolti manageriali che il presente studio offre sono utili a delineare strategie di sviluppo e valorizzazione delle piccole e medie imprese nel contesto europeo, contribuendo al dibattito sulla necessità di un approccio alla pianificazione di marketing e comunicazione nelle PMI. Infatti, questo lavoro fornisce spunti di riflessione utili agli studiosi e ai manager delle PMI che monitorando alcune leve del marketing mix potrebbero assicurarsi un vantaggio competitivo duraturo e difficilmente imitabile.

I limiti della ricerca sono relativi alla ridotta numerosità dei due campioni di imprese assunti a base dell'analisi comparativa, che riduce la possibilità di generalizzazione dei risultati conseguiti. Inoltre, l'eterogeneità dei due campioni rappresenta un ulteriore limite.

Le future ricerche in materia potranno beneficiare di un'estensione dell'indagine ad altri Paesi e dell'impiego di campioni caratterizzati da una maggiore numerosità e rappresentatività. 


\section{Appendice: il questionario somministrato alle imprese}

\section{Anagrafica aziendale}

Ragione sociale:

anno di fondazione:

settore d'appartenenza:

numero di dipendenti:

tipo di prodotto o servizio reso:

target aziendale: fatturato

quota di mercato detenuta nel settore di appartenenza:

1) Qual è il numero di dipendenti impiegati nell'unità organizzativa dedicata alla gestione del marketing e della comunicazione? (indicare il numero)

2)Quali sono i ruoli ricoperti nell'unità organizzativa dedicata alla gestione del marketing e della comunicazione

(specificare)?

3) Quali sono i principali obiettivi svolti dal marketing e dalla comunicazione nella vostra impresa?

(max 3 risposte)

$\square$ aumentare le vendite e migliorare la quota di mercato

口 soddisfare i clienti e promuovere la fedeltà

valutare l'opportunità di entrare in nuovi mercati

口 migliorare l'immagine aziendale (visibilità e notorietà dell'azienda nell'immediato)

口 sviluppare la reputazione (essere giudicati positivamente dai diversi pubblici)

口 promuovere legittimazione e consenso aziendale (farsi conoscere e accettare dalla comunità locale)

D promuovere la coesione interna e il senso di appartenenza del personale

$\square$ selezionare personale qualificato

nessun contributo

non so

altro

(specificare)................................................................................ funzione marketing/comunicazione. Tra i 2 aggettivi ci sono 7 caselle. Se sceglie la 4 , lei ci dice che è neutrale rispetto funzione marketing/comunicazione. Tra i 2 aggettivi ci sono 7 caselle. Se sceglie la 4 , lei ci

ai due aggettivi. Più si muove a sinistra (o a destra), pi

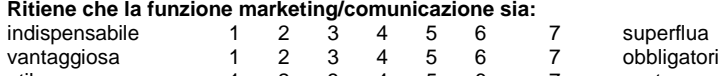

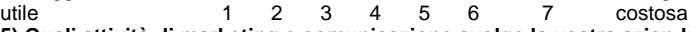

5) Quali attività di marketing e comunicazione svolge la vostra azienda, e in che misura?

\begin{tabular}{|c|c|c|c|c|}
\hline attività di marketing/comunicazione & non svolta & saltuaria & frequente & continuativa \\
\hline segmentazione & $\mathbf{\square}$ & 口 & 口 & $\mathbf{\square}$ \\
\hline targeting & 口 & 口 & $\mathbf{\square}$ & $\mathbf{\square}$ \\
\hline posizionamento & $\bar{\square}$ & $\mathbf{\square}$ & $\mathbf{\square}$ & $\mathbf{\square}$ \\
\hline sviluppo nuovi prodotti & 口 & 口 & 口 & 口 \\
\hline decisioni di packaging & $\mathbf{\square}$ & $\mathbf{\square}$ & 口 & 口 \\
\hline decisioni di prezzo & $\bar{\square}$ & $\overline{\mathbf{D}}$ & $\overline{\mathbf{Z}}$ & $\bar{\square}$ \\
\hline decisioni di distribuzione & 口 & $\mathbf{\square}$ & $\mathbf{\square}$ & $\mathbf{\square}$ \\
\hline decisioni di comunicazione & 口 & 口 & $\square$ & 口 \\
\hline operazioni di branding & प्र & 口 & $\bar{\square}$ & $\bar{\square}$ \\
\hline CRM (customer relationship management) & प्र & प्र & $\bar{\square}$ & प्र \\
\hline ricerche di mercato & 口 & 口 & $\square$ & 口 \\
\hline trade marketing & 口 & 口 & $\mathbf{\square}$ & 口 \\
\hline non so & \multicolumn{4}{|c|}{ 口 } \\
\hline altro (specificare) & & & & \\
\hline
\end{tabular}

6) Quali forme di comunicazione utilizza la sua impresa, e in che misura?

\begin{tabular}{|c|c|c|c|c|}
\hline forme di comunicazione & non utilizza & saltuaria & frequente & continuativa \\
\hline pubblicità (advertising) & 口 & $\mathbf{\square}$ & 口 & 口 \\
\hline $\begin{array}{l}\text { e-advertising (pubblicità web, banner pubblicitari, } \\
\text { social network) }\end{array}$ & 口 & $\bar{\square}$ & $\bar{\square}$ & $\bar{\square}$ \\
\hline sponsorizzazioni & 口 & $\mathbf{\square}$ & $\mathbf{\square}$ & $\mathbf{\square}$ \\
\hline relazioni pubbliche on line & 口 & 口 & 口 & 口 \\
\hline \multicolumn{5}{|l|}{ relazioni pubbliche off line } \\
\hline direct marketing (telemarketing, mailing, etc.) & $\bar{\square}$ & $\bar{\square}$ & $\bar{\square}$ & $\bar{\square}$ \\
\hline promozione vendite & 口 & 口 & 口 & 口 \\
\hline vendita personale & प्र & $\mathbf{\square}$ & $\mathbf{\square}$ & $\mathbf{\square}$ \\
\hline passaparola & 口 & $\bar{\square}$ & $\mathbf{\square}$ & $\mathbf{\square}$ \\
\hline non so & \multicolumn{4}{|c|}{ 它 } \\
\hline altro & & & & \\
\hline
\end{tabular}


7) Quali mezzi di comunicazione utilizza la sua impresa, e in che misura?

\begin{tabular}{|c|c|c|c|c|}
\hline mezzi di comunicazione & non utilizza & saltuaria & frequente & continuativa \\
\hline stampa e redazionali & 口 & $\mathbf{\square}$ & 口 & $\mathbf{\square}$ \\
\hline cartellonistica e affissioni & $\mathbf{\square}$ & $\mathbf{\square}$ & $\mathbf{\square}$ & 口 \\
\hline eventi & 口 & 口 & 口 & 口 \\
\hline televisione & $\overline{\mathbf{D}}$ & $\mathbf{\square}$ & $\mathbf{\square}$ & 口 \\
\hline radio & 口 & $\bar{\square}$ & 口 & $\bar{\square}$ \\
\hline mezzi pubblici (pubblicità dinamica) & $\mathbf{\square}$ & $\mathbf{\square}$ & 口 & 口 \\
\hline internet/new media (sito web, portale, social network) & $\bar{\square}$ & $\bar{Q}$ & 口 & 口 \\
\hline non so & \multicolumn{4}{|c|}{ 口 } \\
\hline
\end{tabular}

8) Quali sono i pubblici destinatari delle sue attività di marketing/comunicazione? (max 2 risposte)

c clienti consumatori

collaboratori interni

Q collaboratori esterni

a azionisti

genti di commercio

$\square$ altri stakeholders (indicare quali)

9) Ricorrete a consulenti esterni per lo svolgimento di attività di marketing/comunicazione?

$$
\mathrm{No}
$$

10) Se SI, a quale tipo di consulente esterno la sua azienda ricorre per le sue attività di marketing/comunicazione?

$\square$ agenzie di pubblicità

agenzie per la creazione siti web

$\square$ consulenti di marketing

$\square$ altre agenzie

non so

$\square$ altro (specificare)

11) Se la sua azienda ricorre a consulenti esterni, lo fa per quale attività di marketing e con quale frequenza?

\begin{tabular}{|c|c|c|c|c|}
\hline attività di marketing & non ricorriamo & ricorriamo raramente & ricorriamo spesso & ricorriamo sempre \\
\hline segmentazione & 口 & 口 & 口 & 口 \\
\hline targeting & प & प & प & प \\
\hline posizionamento & 口 & 口 & 口 & 口 \\
\hline sviluppo nuovi prodotti & 口 & 口 & $\square$ & 口 \\
\hline decisioni di packaging & 口 & 口 & 口 & 口 \\
\hline decisioni di prezzo & 口 & 口 & $\square$ & 口 \\
\hline decisioni di distribuzione & 口 & 口 & 口 & 口 \\
\hline ricerche di mercato & $\bar{\square}$ & $\bar{\square}$ & $\bar{\square}$ & $\bar{\square}$ \\
\hline trade marketing & प्र & $\bar{\square}$ & $\bar{\square}$ & $\bar{\square}$ \\
\hline customer care & 口 & 口 & 口 & 口 \\
\hline CRM & प & प & $\bar{\square}$ & प \\
\hline non so & & & ] & \\
\hline
\end{tabular}

12) Se la sua azienda ricorre a consulenti esterni, lo fa per quale attività di comunicazione e con quale frequenza?

\begin{tabular}{|c|c|c|c|c|}
\hline attività di comunicazione & non ricorriamo & raramente & spesso & sempre \\
\hline pubblicità (advertising) & 口 & 口 & 口 & 口 \\
\hline $\begin{array}{lrll}\text { e-advertising } & \text { (pubblicità } & \text { web, } & \text { banner } \\
\text { pubblicitari, social network) } & & \end{array}$ & $\overline{\mathbf{D}}$ & $\overline{\mathbf{D}}$ & $\bar{\square}$ & प्र \\
\hline sponsorizzazioni & 口 & 口 & 口 & 口 \\
\hline relazioni pubbliche & 口 & $\mathbf{\square}$ & 口 & 口 \\
\hline direct marketing (telemarketing, mailing, etc.) & $\mathbf{\square}$ & $\mathbf{\square}$ & $\mathbf{\square}$ & प \\
\hline promozione vendite & $\mathbf{\square}$ & $\mathbf{\square}$ & 口 & 口 \\
\hline vendita personale & $\mathbf{\square}$ & $\mathbf{\square}$ & $\mathbf{\square}$ & 口 \\
\hline passaparola & 口 & 口 & $\overline{\mathbf{Z}}$ & $\overline{\mathbf{D}}$ \\
\hline stampa e redazionali & 口 & $\mathbf{\square}$ & 口 & 口 \\
\hline cartellonistica e affissioni & प् & $\bar{\square}$ & $\mathbf{\square}$ & 口 \\
\hline eventi & $\mathbf{\square}$ & $\bar{\square}$ & $\mathbf{\square}$ & 口 \\
\hline televisione & $\bar{\square}$ & $\bar{\square}$ & $\bar{\square}$ & $\bar{\square}$ \\
\hline radio & $\mathbf{\square}$ & 口 & $\mathbf{\square}$ & 口 \\
\hline mezzi pubblici (pubblicità dinamica) & $\mathbf{\square}$ & $\mathbf{\square}$ & 口 & 口 \\
\hline sito web (portale) & $\mathbf{\square}$ & 口 & $\mathbf{\square}$ & $\mathbf{\square}$ \\
\hline non so & & 口 & & \\
\hline
\end{tabular}

13) La vostra impresa dispone di un budget destinato alle attività di marketing e comunicazione? No 
14) Se SI in che modo viene calcolato il budget destinato alle attività di marketing e comunicazione?

$\checkmark$ stabiliamo una cifra fissa, indipendentemente dal fatturato

$\square$ percentuale variabile a seconda dei casi

$\square$ percentuale fissa del fatturato

$\checkmark$ lo aggiorniamo rispetto a quello dell'anno precedente

lo stabiliamo in relazione al piano di marketing

$\mathbf{c}$ conformandosi alle tendenze dei competitors

non so

altro (specificare)

15) Rispetto a 2-3 anni fa la sua azienda investe in marketing e comunicazione più o meno di prima?

$\square$ molto meno di prima

$\square$ meno di prima

più o meno come prima

प più di prima

$\square$ molto più di prima

16) Le presentiamo una serie di attività di marketing/comunicazione. Considerando le previsioni di budget della vostra azienda nei prossimi 3 anni, lei dovrà dirci per ogni attività, se l'investimento della sua azienda diminuirà, aumenterà o rimarrà uguale.

17) Quali sono gli strumenti attraverso cui la vostra azienda monitora le attività di marketing e comunicazione?

attraverso ricerche di marketing

\begin{tabular}{|c|c|c|c|}
\hline attività & Diminuirà & Uguale & Aumenterà \\
\hline ricerche di mercato & 口 & 口 & 口 \\
\hline sviluppo prodotti & $\bar{\square}$ & 口 & $\bar{\square}$ \\
\hline analisi distribuzione & प्र & प् & 口 \\
\hline trade marketing & 口 & 口 & 口 \\
\hline analisi prezzi & $\bar{\square}$ & $\bar{\square}$ & $\bar{\square}$ \\
\hline customer care & $\bar{\square}$ & $\bar{\square}$ & $\bar{\square}$ \\
\hline CRM & प्र & प् & प्र \\
\hline stampa e redazionali & 口 & प् & 口 \\
\hline cartellonistica e affissioni & $\bar{\square}$ & $\bar{\square}$ & प्र \\
\hline eventi & $\bar{\square}$ & प् & 口 \\
\hline televisione & 口 & $\bar{\square}$ & 口 \\
\hline radio & $\bar{\square}$ & $\bar{\square}$ & $\bar{\square}$ \\
\hline mezzi pubblici (pubblicità dinamica) & 口 & प् & 口 \\
\hline sito web (portale) & 口 & 口 & 口 \\
\hline non so & & 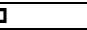 & \\
\hline
\end{tabular}

$\square$ incremento di fatturato a seguito delle attività di marketing/comunicazione

$\mathbf{\square}$ incremento della riconoscibilità del marchio/azienda

$\boldsymbol{\square}$ incremento della reputazione aziendale presso i pubblici di riferimento

non facciamo questo tipo di valutazione

non so

$\square$ altre modalità (specificare)

18) Le presentiamo una serie di affermazioni fatte da alcuni manager delle grandi e piccole imprese. Può indicare, utilizzando la scala prevista, il grado di accordo/disaccordo che attribuisce a ciascuna delle seguenti espressioni.

\begin{tabular}{|l|l|l|l|l|l|}
\hline $\begin{array}{l}\text { La pianificazione di marketing è fondamentale per la } \\
\text { PMI }\end{array}$ & $\begin{array}{c}\text { molto } \\
\text { d'accordo }\end{array}$ & $\begin{array}{c}\text { abbastanza } \\
\text { d'accordo }\end{array}$ & incerto & $\begin{array}{c}\text { poco } \\
\text { d'accordo }\end{array}$ & $\begin{array}{c}\text { per nulla } \\
\text { d'accordo }\end{array}$ \\
\hline $\begin{array}{l}\text { Soltanto le grandi imprese possono ricorrere al } \\
\text { marketing e alla comunicazione }\end{array}$ & & & & & \\
\hline $\begin{array}{l}\text { La pianificazione strategica è un'attività sofisticata, } \\
\text { che può essere svolta esclusivamente dagli } \\
\text { specialisti o dal senior management }\end{array}$ & & & & & \\
\hline $\begin{array}{l}\text { II marketing è superfluo alla crescita e alla } \\
\text { sopravvivenza dell'impresa }\end{array}$ & & & & & \\
\hline $\begin{array}{l}\text { L'impresa che vuole gestire il cambiamento deve } \\
\text { fare leva sulla comunicazione d'impresa }\end{array}$ & & & & & \\
\hline
\end{tabular}

\section{Bibliografia}

BELL J.R., PARKER R.D., HENDON J.R. (2007), "Entrepreneurial application of marketing communication in small business: survey results of small business owners", Entrepreneurial Executive, vol. 12, n. 1, pp. 1-13

BHIDE A. (1994), "How entrepreneurs craft strategies that work", Harvard Business Review, vol. 72 , n. 2, pp. 150-161. 
BROOM H.N., LONGENECKER J., MOORE C.W. (1983), Small Business Management, South Western Publishing Co, Cincinnati, Ohio.

CARSON D. (1985), "The evolution of marketing in small firms", , European Journal of Marketing, Marketing and Small Business (special issue), vol. 19, n. 5, pp. 7-16.

CARSON D., CROMIE S. (1989), "Marketing Planning in Small Enterprises: A Model and Some Empirical Evidence", Journal of Marketing Management, vol. 5, n. 1, pp. 33-49.

CARSON D. (1990), "Some exploratory models for assessing small firms' marketing performance (a qualitative approach)", European Journal of Marketing, vol. 24, n. 11, pp. $25-27$.

CARSON D. (1993), “A philosophy for marketing education in small firms”, Journal of Marketing Management, University of Ulster, vol. 9, n. 2, pp. 189-204.

CARSON D., MCCARTAN-QUINN D. (1995), "Non-practice of theoretically based marketing in small business - issues arising and their implications", Journal of Marketing Theory and Practice, vol. 3, n. 4, pp. 24-31.

CARSON D., CROMIE S., MCGOWAN P., HILL J. (1995), Marketing and Entrepreneurship in SMEs: An Innovative Approach, Prentice Hall, London.

CARSON D., COVIELLO N. (1996), "Qualitative Research Issues at the Marketing/ Entrepreneurship Interface", Marketing Intelligence and Planning, vol. 14, n. 6, pp51-8.

CARSON D., GRANT K. (1998), "SME marketing competence: A definition and some empirical evidence research at the Marketing/Entrepreneurship interface", in Conference proceedings, University of Illinois at Chicago, pp. 173-186.

CARSON D., GILMORE A., CUMMINS D., O'DONNELL A., GRANT K. (1998), "Price setting in SMEs: some empirical findings", Journal of Product and Brand Management, vol. 7, n. 1, pp. 74-86.

CARSON D., GILMORE A. (2000), "Marketing At the Interface: Not 'What' But 'How' ", Journal of Marketing Theory and Practice, vol. 8, n. 2, pp. 1-7.

COHN T., LINDBORE R.A. (1972), "How marketing is different in small companies", An American Management Association Management Briefing, American Management Association.

CORVI E., FIOCCA R. (1996), Comunicazione e valore nelle relazioni aziendali, Egea, Milano.

COZZI G. (1985), "Il marketing nelle piccole e medie imprese", in L'economia delle piccole $e$ medie imprese industriali, Atti del Convegno Aidea, Urbino, Editrice Clueb, Bologna.

DE LUCA A. (2009), Innovazione e competitività delle PMI in Italia. Metodi e Modelli di mercato, Franco Angeli, Milano.

FABRIS G. (2003), La comunicazione d'impresa, dal mix di marketing al communication mix, Sperling § Kupfer Editori, Milano.

FILLIS I. (2002), "Small Firm Marketing Theory and Practice: Insights from the Outside", Journal of Research in Marketing \& Entrepreneurship, vol. 4, n. 2, pp. 134-157.

FERRERO G., FORTEZZA F. (2007), "Importanza ed elementi di criticità del marketing strategico nelle PMI", Piccola Impresa/Small Business, n. 2, pp. 59-89.

GILMORE A., CARSON D., ROCKS S. (2006), "Networking in SMEs: Evaluating its Contribution to Marketing Activity”, International Business Review, vol. 15, n. 3, pp. 278-293.

GOLINELLI G.M. (1992) "I problemi strategici delle imprese minori”, Sinergie, n. 27, pp. 25-31. 
GRANDINETTI R. (1989), "Il marketing delle grandi e delle piccole imprese: dalla separazione alla convergenza", Economia e Politica Industriale, n. 63, pp. 83-120.

GUERCINI S. (2005), "Marketing imprenditoriale, marketing manageriale e conoscenza di mercato del vertice d'impresa, Mercati e Competitività, n. 1, pp. 143-164.

HÄTÖNEN J., RUOKONEN M. (2010), "Revising marketing strategies for supplier selection criteria: small firm approach from the information and communications industry", Journal of Business \& Industrial Marketing, vol. 25, n. 2, pp. 159-167.

HILL J. (2001a), "A Multidimensional Study of the Key Determinants of Effective SME Marketing Activity: Part 1", International Journal of Entrepreneurial Behaviour \& Research, vol. 7, n. 5, pp. 171-204.

HILL J. (2001b), "A Multidimensional Study of the Key Determinants of Effective SME Marketing Activity: Part 2", International Journal of Entrepreneurial Behaviour \& Research, vol. 7, n. 6, pp. 211-235.

HOGARTH-SCOTT S., WATSON K., WILSON N. (1996), "Do small businesses have to practice marketing to survive and grow?", Marketing Intelligence \& Planning, vol. 14, n. 1, pp. 6-18.

KIRBY D.A., TRAVIS L. (1995), "Marketing and the Small Service business: Solecitors practices in England", Research at the Marketing/Entrepreneurship Interface, University of Illinois at Chicago, Conference proceedings, pp. 71-94.

KITCHEN P.J., SCHULTZ D.E. (2000), "A response to theoretical concept or management fashion", Journal of Advertising Research, vol. 40, n. 5, pp. 17-21.

INVERNIZZI E., ROMENTI S. (2013), Relazioni pubbliche e corporate communication. Le competenze e i servizi di base, vol. 1, McGraw-Hill, Milano.

INVERNIZZI E., ROMENTI S. (2012), Relazioni pubbliche e corporate communication. vol. 2, McGraw-Hill, Milano.

LILIEN G.L., KOTLER P., MOORTHY K.S. (1992), Marketing Models, Prentice-Hall, Englewood Cliffs, NJ.

MARCHINI I. (1985), "Attualità, specificità e strategie delle piccole e medie imprese. L'economia delle piccole e medie imprese industriali”, Atti del Convegno Aidea, Urbino, Editrice Clueb, Bologna.

MATTIACCI A., CECCOTTI F. (2005), "Lo sviluppo del marketing nella PMI: riflessioni a margine di un'esperienza d'impresa", Micro \& Macro marketing, vol. 14, n. 1, pp. 65106.

MAURI C. (2011), Marketing per le PMI, Egea, Milano.

MAURI C. (1997), "Quale marketing per le piccole e medie imprese?", in Brunetti G., Musatti G., Corbetta G., Piccole e medie imprese e politiche di facilitazione, Egea, Milano, pp. 243-254.

MCCARTAN-QUINN, D., CARSON, D. (2003), "Issues which impact upon marketing in the small firm, Small Business Economics, vol. 21, n. 2, pp. 201-213.

MENDHAM S., BANNOCK G. (1982), "Small business and economic change". Paper presented at the International Congress on Small Business, October, Malaga, Spain.

O'DWYER M. (2009), Marketing the SME. Innovation and Approach, Cambridge Scholars Publishing.

O'DWYER M., GILMORE A., CARSON D. (2009), "Innovative marketing in SMEs", European Journal of Marketing, vol. 43, n .1/2, pp. 46-61.

PACITTO J.C., BIZEUL P.A.J., BIZEUL P. (2007), "Marketing in medium-sized manufacturing firms: The state-of-the-art in France and in Quebec", International Entrepreneurship Management Journal, vol. 3, n. 1, pp. 29-50. 
PASTORE A., MATTIACCI A. ( 2013), Marketing. Il management orientato al mercato, Hoepli, Milano.

PENCARELLI T., CIOPPI M. (2006), "I processi di vendita e il marketing nelle PMI", paper presentato al Convegno di Parma della Società Italiana di Marketing, Parma, 24-25 Novembre 2006, pp.1- 30.

REYNOLDS P.L. (2002), "The Need for a New Paradigm for Small Business Marketing? What is Wrong with the Old One?", Journal of research in marketing and entrepreneurship, vol. 4, n. 3, pp. 191-205.

SCASE R., GOFFEE R. (1987), The Real World of the Business Owner, Croom Helm, London.

SCHOLLHAMMER H., KURILOFF A. (1979), Entrepreneurship and Small Business Management, John Wiley, New York.

SIANO A., VOLLERO A., SIGLIOCCOLO M. (2013), "Corporate communication management: A framework based on decision making with reference to communication resources", Journal of Marketing Communications, vol. 19, n. 3, pp. 151-167.

SIMPSON M., TAYLOR N. (2002), 'The Role and Relevance of Marketing in SMEs: towards a new model', Journal of Small Business and Enterprise Development, vol. 9, n. 4 , pp. $370-382$.

SIU W., KIRBY D.A. (1998), “Approaches to Small Firm Marketing. A Critique”, European Journal of Marketing, vol. 32, n. 1/2, pp. 40-60.

STEINHOFF D. (1978), Small Business Management Fundamentals, McGraw-Hill, Maidenhead.

THRASSOU A., VRONTIS D. (2006), “A Small Services Firm Marketing Communications Model for SME-Dominated Environments", Journal of Marketing Communications, vol. 12, n. 3, pp. 183-202.

VARALDO R. (1983), "L'approccio di marketing nelle piccole e medie imprese”, in atti del seminario sulle "Strategie di sviluppo delle piccole e medie imprese", 11 giugno, Ancona.

VECCHIATO G. (2005), Relazioni Pubbliche: valore che crea valore, Franco Angeli, Milano.

VESCOVI T., GAZZOLA P. (2006), "Strutture e uso degli strumenti di marketing e comunicazione nelle imprese del Regno Unito. Primi risultati di una ricerca europea", Atti del $\mathrm{V}^{\circ}$ Convegno Internazionale, Le tendenze del Marketing in Europa, Venezia, 20-21 Gennaio 2006, pp. 1-20.

WATZLAWICK P., BRAVIN J.H., JACKSON D.D. (1971), Pragmatica della comunicazione umana, Astrolabio, Roma.

WONG H.Y., MERRILEES B. (2008), "Determinants of SME international marketing communication, Journal of Global Marketing, vol. 21, n. 4, pp. 293-305. 